For Publisher's use

\title{
QUANTUM CHROMODYNAMICS AT COLLIDERS
}

\author{
J. M. BUTTERWORTH \\ Department of Physics \& Astronomy, University College London, WC1E 6BT, London, UK \\ E-mail: J.Butterworth@ucl.ac.uk
}

\begin{abstract}
QCD is the accepted (that is, the effective) theory of the strong interaction; studies at colliders are no longer designed to establish this. Such studies can now be divided into two categories. The first involves the identification of observables which can be both measured and predicted at the level of a few percent. Such studies parallel those of the electroweak sector over the past fifteen years, and deviations from expectations would be a sign of new physics. These observables provide a firm "place to stand" from which to extend our understanding. This links to the second category of study, where one deliberately moves to regions in which the usual theoretical tools fail; here new approximations in QCD are developed to increase our portfolio of understood processes, and hence our sensitivity to new physics. Recent progress in both these aspects of QCD at colliders is discussed.
\end{abstract}

\section{The Data and the Experiments}

QCD studies at colliders involve measurements of the hadronic final state in $e^{+} e^{-}$, lepton-hadron and hadron-hadron collisions. The lepton colliders also allow the study of effective photon-photon, lepton-photon and photon-hadron collisions, due to the almoston-shell photon beam which accompanies lepton beams. In collisions involving these photons, the photon may participate directly in the hard process, or it may act as a source of partons much like a hadron. Together, this array of different colliding beams provides us with many data and rich opportunities to learn from cross-comparison between experiments.

Data presented at this meeting include precise measurements of a great number of properties of the final state, and these measurement are used to demonstrate and improve our understanding of the physics. With the confidence that this is understood, it then becomes possible to infer, from an increasing number of measurements, information about the initial state; that is, quarks and gluons in their natural habitat inside hadrons. This in turn enables us to predict effects at future colliders, particularly the Large Hadron Collider under construction at CERN ${ }^{1}$.

In sections $2-5$, the final state mea- surements are discussed. In the subsequent section, some experimental advances in the current knowledge of parton densities within the Dokshitzer-Gribov-LipatovAltarelli-Parisi (DGLAP) paradigm are presented. Following that, some measurements in regions of phase space where DGLAP evolution is not applicable are discussed. This includes low $x$ and diffractive effects, at which point I conclude this contribution and hand over to the next speaker ${ }^{2}$.

\section{Fragmentation and Hadron Production}

An obvious observable to start with in looking at QCD final states is the charged particle multiplicity. This has been measured as a function of the energy scale of the interaction by many experiments. A summary ${ }^{3}$ is shown in Fig. 1. The energy scale dependence is seen to be universal to within a few percent for reasonable definitions of the energy scale in $e^{+} e^{-}$and DIS, and the proton data from ISR also lies close to the same curve. This is well modelled by the current Monte Carlo (MC) models. The shape is also described by next-to-leading-order (NLO) QCD (not shown), where local parton-hadron duality is assumed to give an arbitrary constant normalisation factor. 


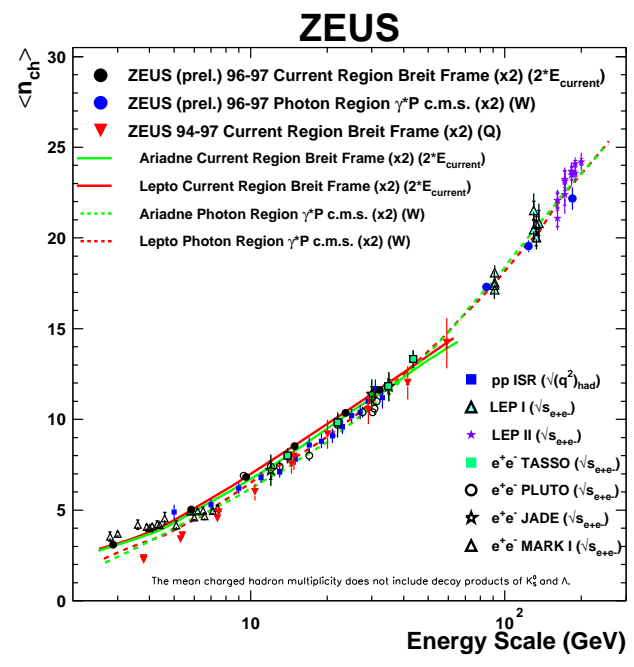

Figure 1. The charged particle multiplicity as a function of energy scale for a selection of experiments.

To make more precise statements about QCD fragmentation, measurements can be designed specifically to suit precise calculations. Accurate calculations for quark and gluon fragmentation exist for hemispheres of a fragmenting diquark of di-gluon system. In the case of quarks, this is a natural configuration for comparison with $e^{+} e^{-}$data. Obtaining a comparable configuration for gluons, however, is more difficult. In a contribution from $\mathrm{OPAL}^{4}$ the jet boost algorithm is employed to do this. Precise agreement is observed for $0.06<x<0.8$. Because of this level of agreement, fundamental parameters of the theory can be extracted with confidence. An impressive recent example is the measurement of the ration of the gluon and quark colour factors, $C_{A} / C_{F}=$ $2.261 \pm 0.014 \pm 0.036 \pm 0.066$, by DELPHI ${ }^{5}$, where the first error is statistical, the second the experimental systematic error and the the third the theoretical uncertainty. This agrees well with the QCD expectation of 2.25 .

One assumption employed in such measurements is that the soft, hadronization stage can be controlled and seperated from
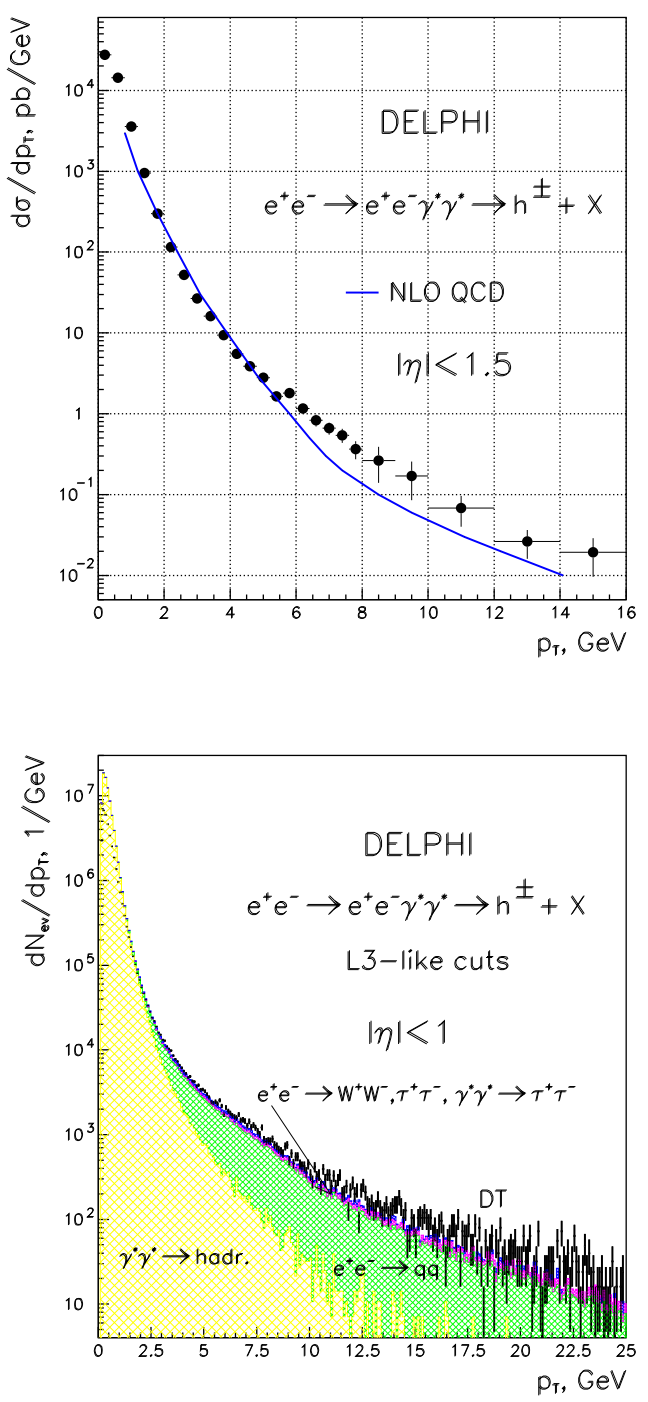

Figure 2. The charged particle cross section in $\gamma \gamma$ collisions as a function of particle transverse momentuym $\left(p_{T}\right)$ as measured by DELPHI. The upper plot is the DELPHI measurement of the cross section compared to NLO QCD. The lower plot is the DELPHI data analysed using cuts close to those used by L3 (see text). 
the hard QCD process. This assumption has been tested in many measurements, and several new results from HERA ${ }^{6}$ have tested it in the case of charm quarks. Here it has been shown that the fraction of charm quarks fragmenting to the various charmed hadrons is the same (to within the measurement accuracy of a few \%) in DIS and photoproduction at HERA as it is in $e^{+} e^{-}$annihilation. Comparisons between the fragmentation function at HERA, LEP and CLEO also show qualitative agreement. A fit of the fragmentation function using NLO calculations would allow a more quantitative statement to be made here, and would be of great interest; as would more accurate measurements from HERA II.

The claim is that for some QCD observables the theoretical understanding is so good that deviations in the data really do mean new physics. This claim was challenged by two results from the L3 collaboration, where in $\gamma \gamma$ events, both the charged particle and jet cross sections lie above the NLO QCD prediction, with a discrepancy which increases as the scale increases ${ }^{7}$. This discrepancy seems impossible to reconcile with $\mathrm{QCD}$; yet the scale is so low $\left(p_{T} \approx 5 \mathrm{GeV}\right.$ for the charged hadrons) that some beyond-thestandard-model explanation seems unlikely. The charged particle measurement has been repeated by $\mathrm{DELPHI}^{8}$, however, and no such discrepancy is seen (Fig.2 - note that no theoretical uncertainty is shown). To their great credit, DELPHI have gone further, solving the puzzle by mimicking the L3 analysis and showing that for the L3 selection cuts there is a large background from annihilation, which has the correct charactierstics to explain the discrepancy. This is also shown in Fig.2; it is then a victory for some kind of precision QCD. It is tempting to speculate that the $e^{+} e^{-}$background may also contribute to the excess seen by L3 in the jet cross section.

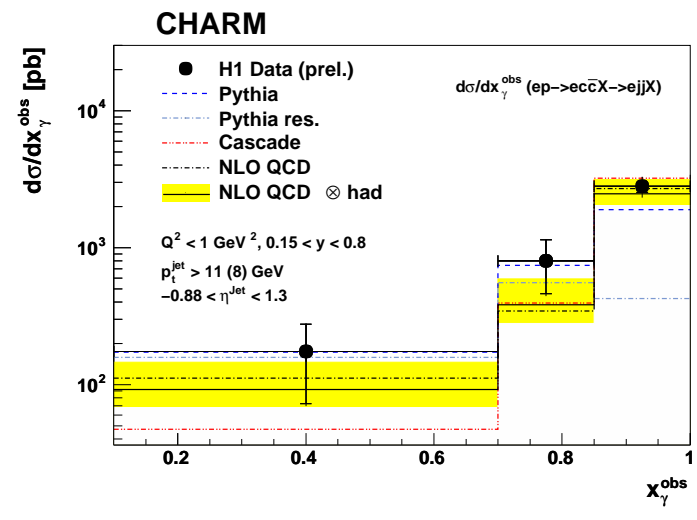

Figure 3. The $x_{\gamma}^{\mathrm{OBS}}$ distribution in charm photoproduction.

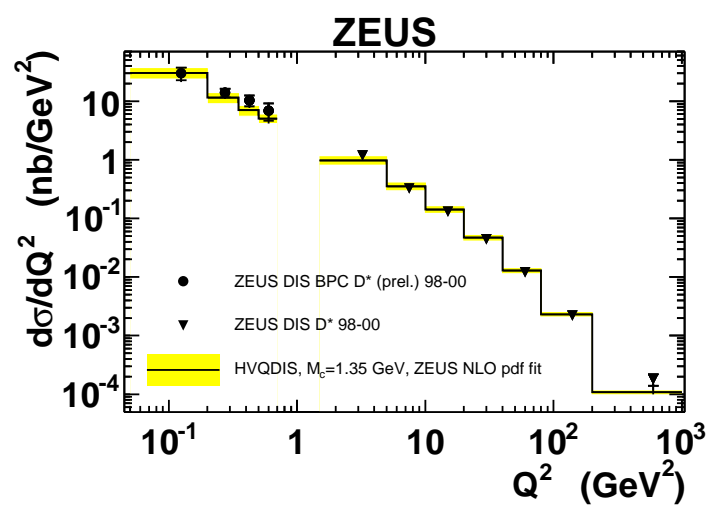

Figure 4. The inclusive charm cross section as a function of $\mathrm{Q}^{2}$.

\section{Charm and Beauty Production}

Recent data on fragmentation properties of charm have been briefly discussed above. The production cross sections for both charm and bottom quarks also represent an important investigative tool for QCD, and since bottom in particular is often used as a tag in searches for new physics, the QCD production mechanism is of particular importance. An understanding of the production dynamics as well as inclusive rates is needed. Results continue to be produced from $p \bar{p}, e p$ DIS and photoproduction. 


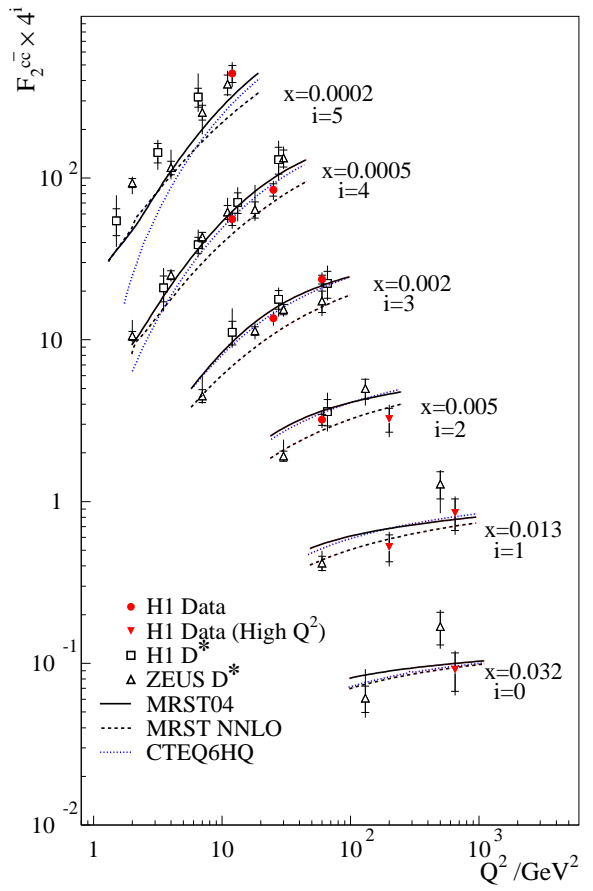

Figure 5. The charm tagged structure function of the proton.

\subsection{Charm cross sections}

Photoproduction of charm has been measured using tagged $D^{*}+$ jets and via lifetime tagging ${ }^{9,10,11}$. Changing the fraction of the photon's momentum seen in the jets, $x_{\gamma}^{\mathrm{OBS}}$, from values near one to lower values allows one to move from so-called direct processes, dominated by point-like photons, to resolved processes, in which the photon acts as a source of partons similar to a hadron. Both regions are well described by NLO QCD calculations (Fig.3). In addition, the inclusive cross section is well understood in both the photoproduction and DIS regimes, from photon virtualities of near zero up to $1000 \mathrm{GeV}^{2}$ (Fig. 4). Expressed as the charm structure function $F_{2}^{\mathrm{c} \overline{\mathrm{c}}}$, the data is already quite precise and is still being accumulated. Again, NLO QCD describes it well (Fig. 5) ${ }^{12}$.

On a related topic, inelastic $J / \psi$ production, the debate about colout octet terms is not yet resolved. NLO QCD corrections to the colour singlet term are very large ${ }^{11}$.

\subsection{Beauty cross sections}

Inclusive measurements of bottom-tagged cone dijets from the CDF II have been measured $^{17}$ and compared to PүтніА ${ }^{13}$, Herwig $^{14}$ and MC@NLO ${ }^{15}$ (Fig. 6a). The normalisation of the LO MCs has a large uncertainty associated with it due to higher order terms. However, it is significant that PүтніA describes the shape of the data very well for $E_{T}^{\text {jet }}>40 \mathrm{GeV}$. $\mathrm{MC} @ \mathrm{NLO}$ is in good agreement with the cross section at high transverse momenta but falls below the data at $E_{T}^{\text {jet }}<70 \mathrm{GeV}$. Apart from the NLO terms, one difference between the two programs is that PYTHIA includes a multiparton interaction model to describe the underlying event. Adding such a model to MC@NLO in the shape of JimmY ${ }^{16}$, leads to good agreement between MC@NLO and the data for $E_{T}^{\text {jet }}>40 \mathrm{GeV}$ (Fig. 6b).

There are also measurements from D0 of muon-tagged jets ${ }^{18}$, where within $50 \%$ errors NLO calculations describe the data. At HERA, DIS and direct photoproduction measurements are reasonably well described, though there is a tendency for the data to be above the calculations. This seems particularly pronounced at low $x_{\gamma}^{\mathrm{OBS}}$ (see Fig.7), where it is possible that non-perturbative effects such as the underlying event may play some role. Precision data from HERA II will hopefully clarify the situation.

Finally, the first measurements of the beauty stucture function $F_{2}^{\mathrm{b} \overline{\mathrm{b}}}$ have now been made ${ }^{12}$, shown in Fig. 8. These lag the similar charm measurements in statistical precision, but there are many more data to come, and it will be an important challenge for the theory to describe such inclusive measurements well. 

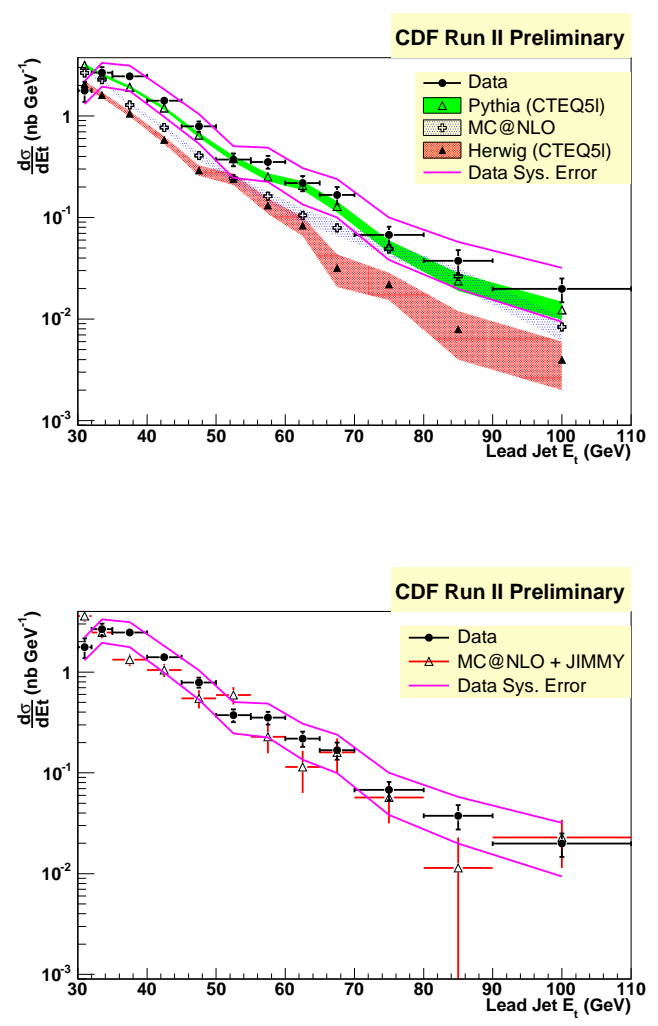

Figure 6. Bottom-quark jet cross sections from CDF II.

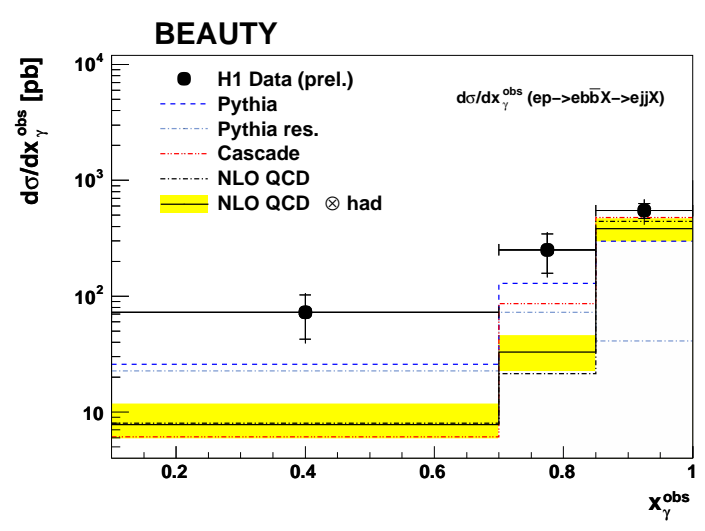

Figure 7. The $x_{\gamma}^{\mathrm{OBS}}$ distribution in bottom photoproduction.

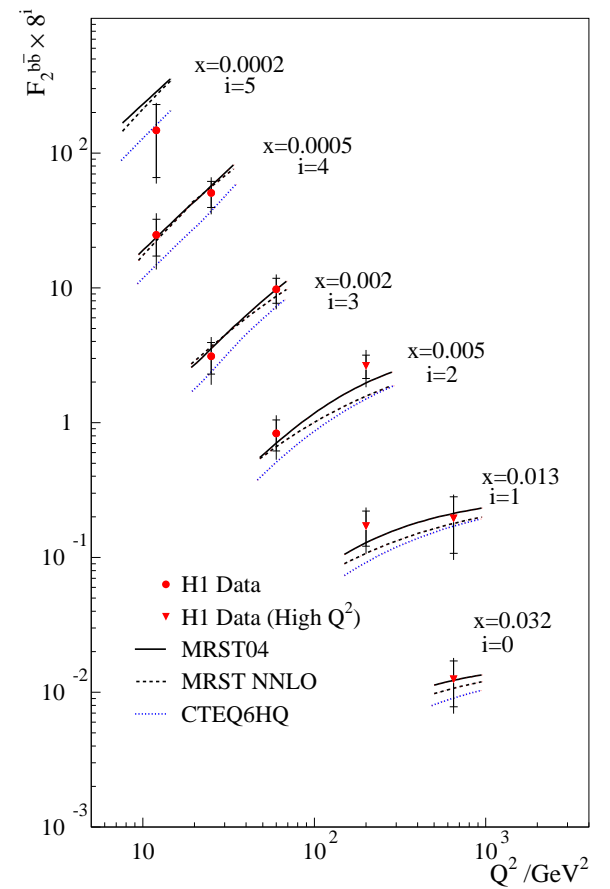

Figure 8. The bottom-quark tagged structure of the proton.

\subsection{Charm and Bottom production dynamics}

The charm statistics at HERA are sufficient that the production dynamics may be measured. Several measurements already exist ${ }^{11}$, and there are new measurements now of the azimuthal correlation of dijets in charm events ${ }^{10}$, as well as jet shapes for charm jets ${ }^{9}$. Both are sensitive to $\mathrm{QCD}$ radiation in these processes. The azimuthal decorrelation is well described by leading-logarithmic parton shower models for both resolved and direct photoproduction; NLO calculations for massive charm quarks (e.g. in which the charm is not an active quark in the photon or proton) describe the direct case well, but fail to describe the low- $x_{\gamma}^{\mathrm{OBS}}$ decorrelation (see Fig.9. The jet shapes are well described by PYTHIA's parton showers for high $x_{\gamma}^{\text {OBS }}$, but the jets are narrower in the data than in the $\mathrm{MC}$ at low $x_{\gamma}^{\mathrm{OBS}}$. 


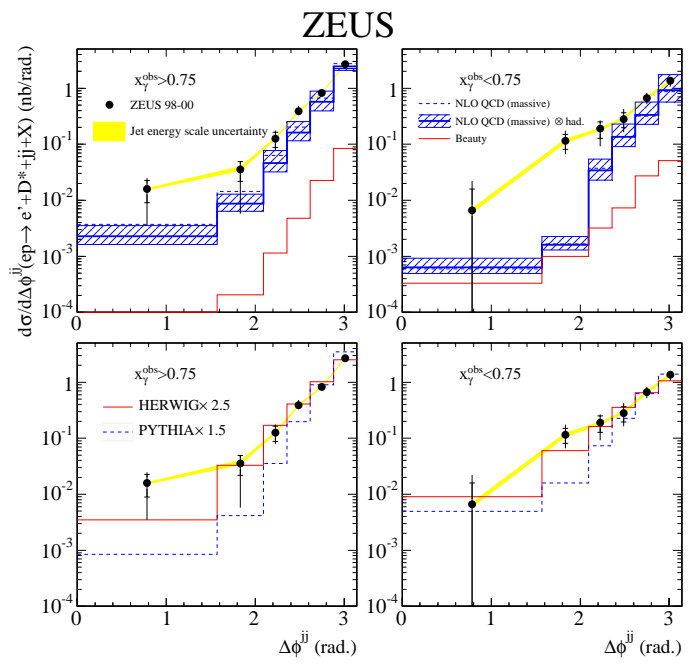

Figure 9. The $\Delta \phi$ distribution for charm jets.

In the case of beauty, the Tevatron data allow studies of such properties in bottom quark events. The dijet correlation is reasonably well described by MC@NLO, but the addition of multiparton interactions does again improve the agreement. PythiA also does a reasonable job.

Finally, a beautiful new measurement of the ratio of bottom- to light-quark jet rates from DELPHI ${ }^{19}$ leads to an accurate measurement of the running b-mass $m_{b}(Q)=$ $4.25 \pm 0.11 \mathrm{GeV}$ at threshold.

In summary of this section, it does seem that in general charm and bottom production are well described by NLO QCD, but that there is a need to combine state-of-art nonperturbative models with the best perturbative calculations in order to get this level of agreement. This is true particularly for measurements in hadronic collisions spanning a large range in transverse energy.

\section{Jet Structure and Event Shapes}

Measurements of jet cross sections and event shapes continue to improve in precision, as do calculations of such properties. This means

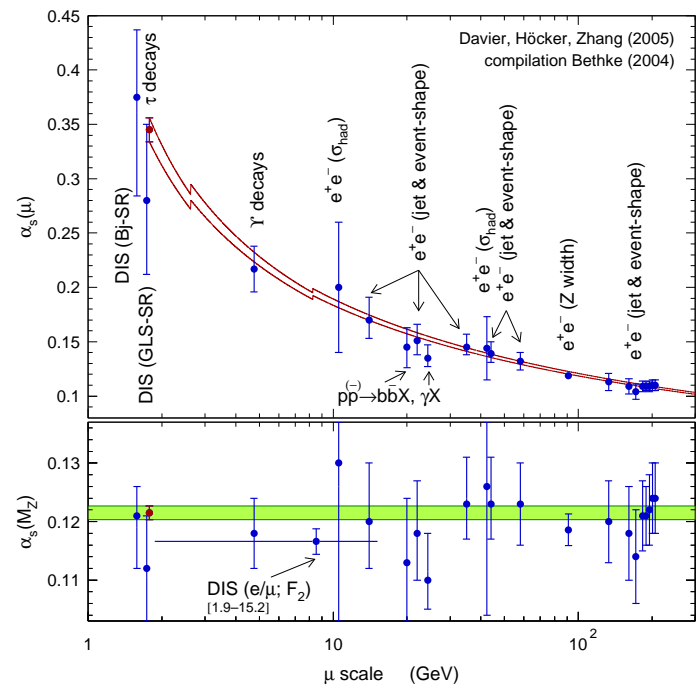

Figure 10. A selection of $\alpha_{s}$ measurements.

that the strong coupling, $\alpha_{s}$, may be extracted from a large number of final states in many processes. At this conference, new results from $e^{+} e^{-}$(JADE, OPAL, ALEPH) and $e p(\mathrm{H} 1, \mathrm{ZEUS})$ were presented ${ }^{20,21}$. A particularly interesting measurement is the ALEPH extraction from $\tau$ decays, shown in Fig. 10, which greatly improves the accuracy at low scales ${ }^{21}$. In general, none of the others is a great leap forward in itself, but all steadily improve accuracy of the world average, and build confidence in our understanding of QCD.

Behind this achievement lies an increasing number of well-understood QCD processes. Perhaps particularly noteworthy this year are the new inclusive jet measurements from Tevatron Run II and HERA, where the use of well-controlled jet algorithms and the impressive level of knowledge of the energy scale and resolution in the experiments means that the data really lay down a strong challenge for the theoretical predictions. Some of the CDF II results are shown Fig. 11; here the $K_{\perp}$ algorithm has been used with different distance parameters; this is an important technique, in that any new physics ef- 
fect seen in such cross sections should be present for all reasonable choices, whereas the sensitivites to some non-perturbative effects will vary between different algorithms and parameters. Another interesting process with new data is prompt photon production, where both HERA and Tevatron have new data $^{22,23}$. The D0 data in particular now show impressive agreement with QCD over a wide range of transverse energy.

\section{Production of jets with bosons}

When the LHC starts delivering data, an unprecedented number of $W$ and $Z$ particles will be produced, usually in association with jets. They feature in many "standard candle" cross sections which will be used to extract parton densities and calibrate the detectors, as well as in many exotic signatures for new physics. It is imperative to understand as far as possible equivalent processes at existing colliders, particularly the Tevatron. The dijet correlation ${ }^{24}$ at D0 is shown in Fig 12. It is well described by NLO QCD in the important wide-angle area where the fixed-order tree-level diagrams are most significant, and is described by parton shower MC in the low angle regions, as expected. Importantly, the SHERPA program matches these two types of calculations and describes the whole shape well ${ }^{25}$.

A related cross section is the diphoton decorrelation, measured by $\mathrm{CDF}^{23}$, shown in Fig. 13. The angle between the two photons is well described by NLO QCD as contained in the DIPHOX ${ }^{26}$ program. The RESBOS ${ }^{27}$ calculation does not include NLO fragmentation contributions and falls below the data at high angles.

Run II measurements of $Z$ cross sections are now coming out, and both the inculsive $Z$ rapidity ${ }^{28}$ and the $N$-jet rate in $Z$ events $^{29}$ are in good agreement with NLO QCD (Fig.14).
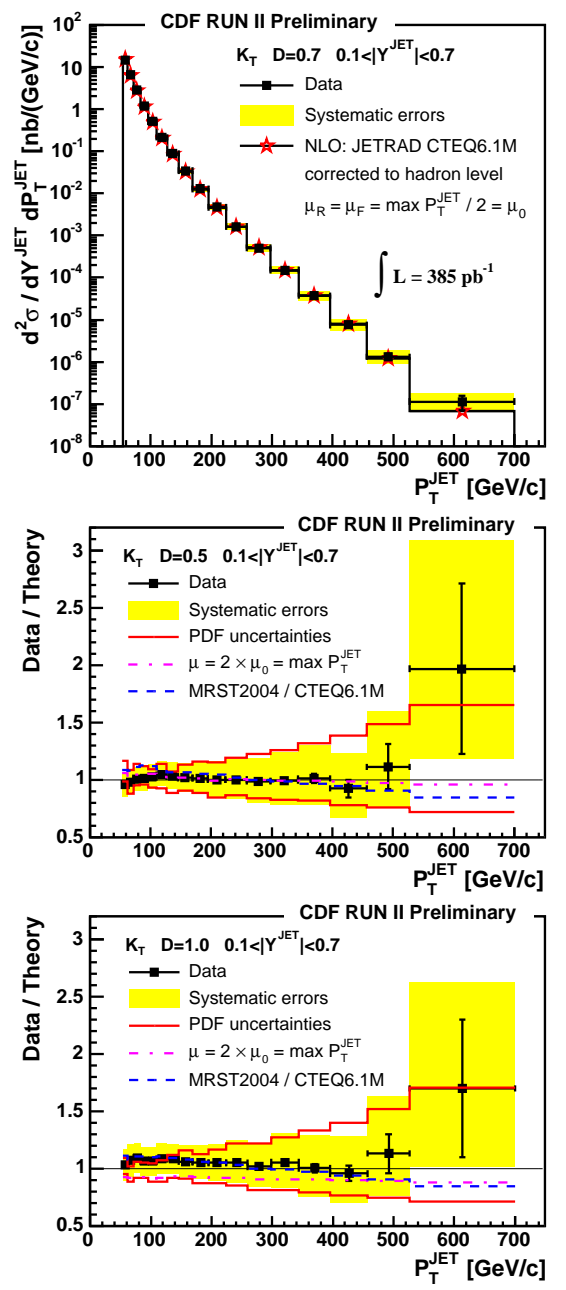

Figure 11. CDF inclusive jet measurements using the $K_{\perp}$ algorithm. The top plot shows the measured differential cross section $d \sigma / d p_{T}^{\text {jet }}$ compared to NLO $\mathrm{QCD}$ for $R=0.7$. The lower two plots show the ratio of data/theory for similar cross sections measured with $R=0.5$ and $R=1.0$. 


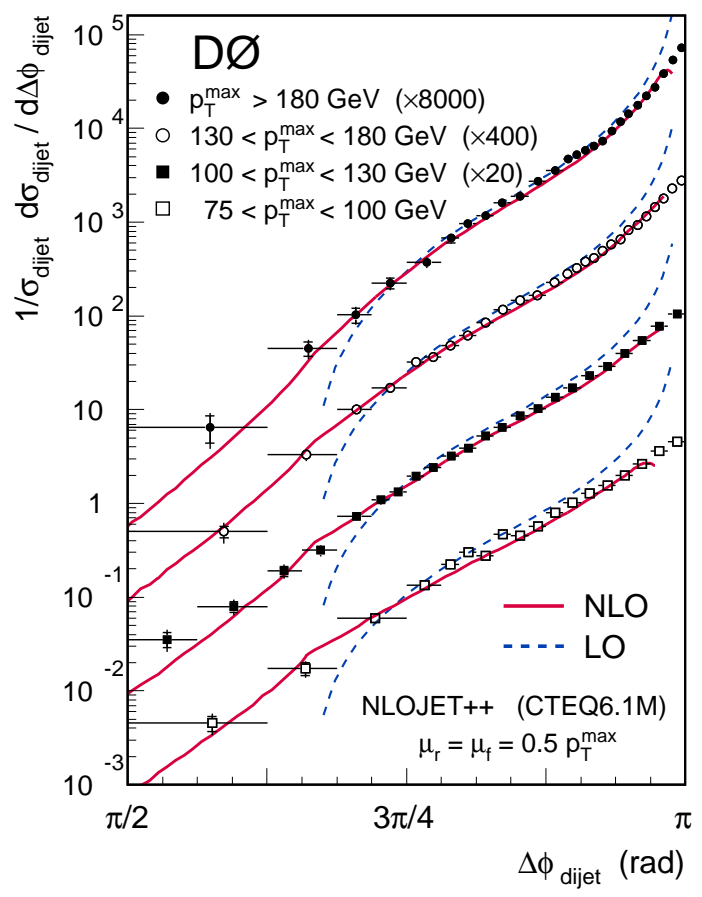

Figure 12. Dijet decorrelation from D0.

\section{Parton Densities}

There has been major theoretical progress in this area, as discussed in the previous contribution $^{30}$. There have also been some notable experimental advances, which are discussed below.

\subsection{High $x$}

The kinematic plane at the LHC is shown in Fig. 15, along with the regions where LHC and other data will be able to constrain the gluon density in the proton. There is an urgent need more information about the gluon at high $x$ (say 0.05 and above) and at $\mathrm{Q}^{2}$ between 100 and $10000 \mathrm{GeV}^{2}$, so that reliable predictions may be made for the highest energy cross sections at LHC. In addition there is a strong correlation between $\alpha_{s}$ and the gluon for intermediate $x$ values ( 0.001 to $0.05)$ in fits to $F_{2}$.

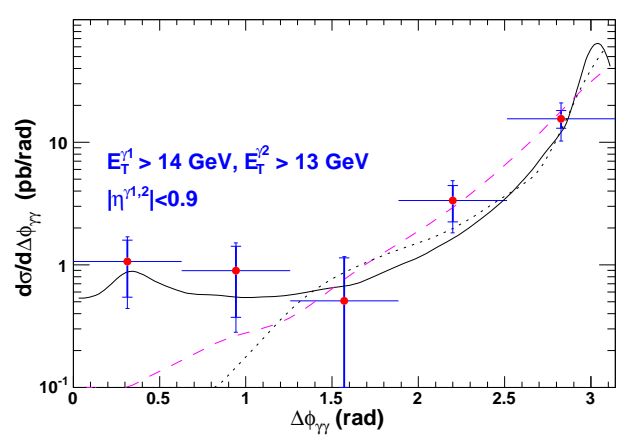

Figure 13. Diphoton decorrelation from CDF. The points are the data, the solid line is the DIPHOX calculation and the dashed is RESBOS (see text).

Including DIS jet cross sections in the fit constrains the coupling, but these cross sections are dominantly quark initiated and depend only weakly on the gluon density. Jet photoproduction, on the other hand, is dominantly gluon initiated over a wide kinematic range, as can reach very high $x$. ZEUS have included both in a $\mathrm{fit}^{35}$, with their latest inclusive cross section data, and see a significant improvement in the accuracy of both $\alpha_{s}$ and the gluon at high $x$. Perhaps most excitingly, the jet data used was a fraction (around a tenth) of the total expected by the end of HERA II. There are major improvements expected ${ }^{36}$.

HERA II is also now producing high luminosities of electron-proton collisions (rather than positron-proton), and early measurements were shown at this conference. The large increase of statistics, matching or bettering that achieved with positrons, and coupled with lepton polarization, brings several benefits. One is the ability to measure the electroweak structure of quark coupling (see a previous contribution ${ }^{31}$ ). The measurement of charged and neutral currents will also allow constraints on flavour composition of proton to be made from HERA data alone, avoiding nuclear correction uncertainties from fixed target data. These data also 


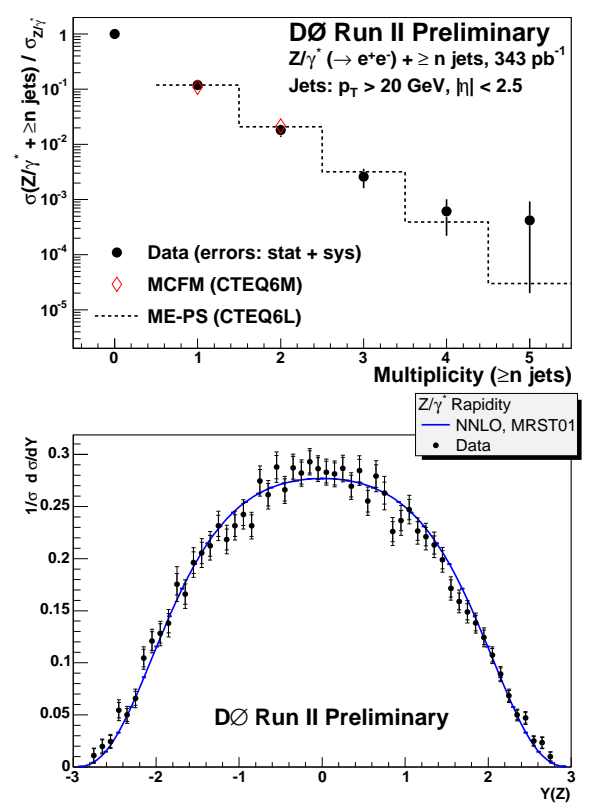

Figure 14. The $N$-jet rate in $Z$ events, compared to $\mathrm{MC}$ calculation (upper plot) and the $Z$ rapidity distribution (lower plot).

reach up to high $x$.

At lower $\mathrm{Q}^{2}$ it is still in principle possible to reach high $x$, since the scattered electron may be measured. However, the radiative corrections are such in this region that while reconstruction of $\mathrm{Q}^{2}$ from electron is good, it is very poor for $x$. A new measurement from ZEUS $^{32}$ uses the hadronic jet to reconstruct $x$. As $x$ increases, the jet moves forward and will at some point be lost down the forward beampipe. However, in this case it is possible to set a minimum $x$ based on the fact that the hadronic jet escaped, and integrate above this. The measurement gives a good sensitivity to the high $x$ structure function, as shown in Fig. 16.

Finally in this subsection, the $W$ asymmetry measurements from tevatron run II are now appearing ${ }^{33}$. They are sensitive to flavour composition in proton at high $x$ and will be important input to new fits.

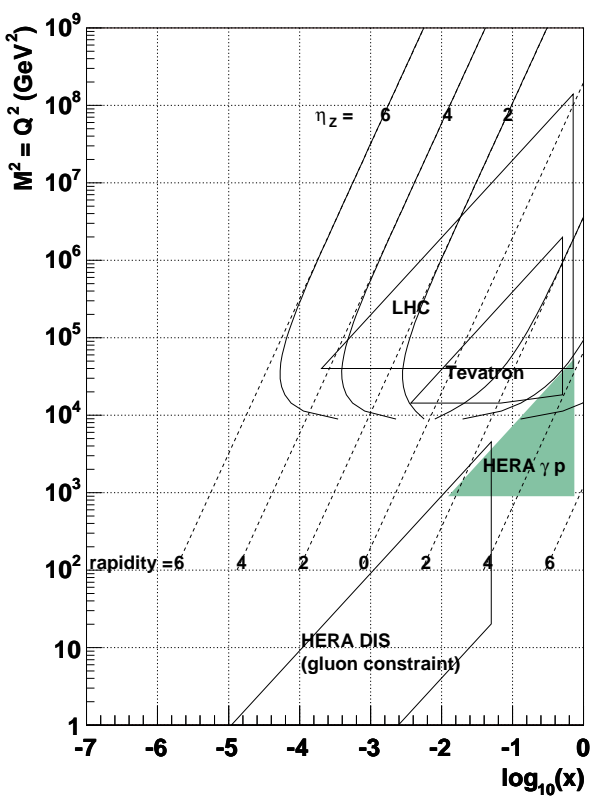

Figure 15. The parton kinematics at LHC and elsewhere. The curved lines show the region where $Z+$ jet production might be used to constrain the gluon at the LHC.The HERA and Tevatron regions shown are those where the gluon may be constrained from $F_{2}$ fits and jet production.

\subsection{Low $Q^{2}$}

Measuring inclusive lepton-proton crosssections in the low $\mathrm{Q}^{2}$ region probes the transition from a region where perturbative calculations are valid to a region where non-perturbative techniques must be used to make any prediction. It also provides the lowest reach in $x$, and thus sensitivity to high density QCD. Two new measurements from H1 have been presented in this area ${ }^{34}$. In the first, QED Compton events, with a high virtuality exchanged electron, are used. In this case the electron virtuality means that the final state electron can be detected even when the virtuality of the exchanged photon is very low. In the second such measurement, initial state photon radiation is tagged, which implies a low virtuality incoming electron with an energy lower than the beam energy. This incoming electron energy is measured from the longitudinal energy imbalance in the cen- 


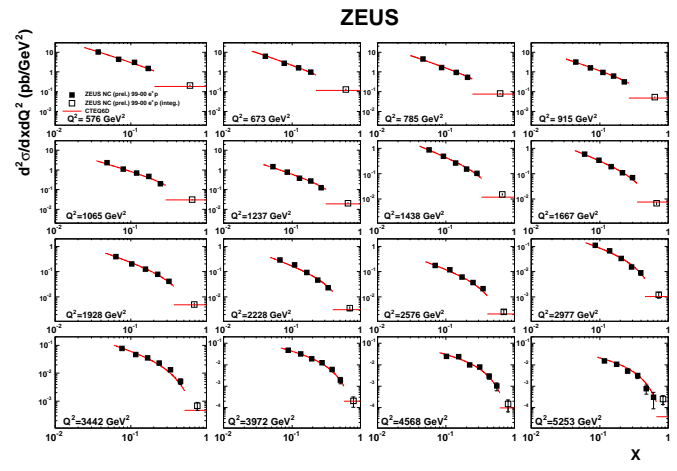

Figure 16. New measurements of $F_{2}$ at high $x$.

tral detector. This allows the measurement to be made at lower $\mathrm{Q}^{2}$ while keeping $x$ moderately high. Both of these measurements provide new data in the transition region between DIS and photoproduction.

\section{Peripheral Collisions, Low $\mathrm{x}$ and Diffraction}

The low $\mathrm{Q}^{2}$ region discussed above is an example of a measurement where we deliberately extend into a region where the usual theoretical tools are expected to fail. Moving into such regions allow the investigation of new approximations in QCD such as clever resummations, new evolution equations, new perturbative expansions, high parton densities and correlated parton distributions. Using the data to verify or falsify such tools extends our portfolio of understood QCD phenomena. There is a large overlap in this area with both the previous ${ }^{30}$ and following ${ }^{2}$ speakers, and I will concentrate on the topics least aligned with theirs.

\subsection{New resummations and evolutions}

The parton density fits discussed above all use the DGLAP evolution equations, which are strongly ordered in the scale, $Q_{1} \gg Q_{2} \gg$ $Q_{3}$. For inclusive properties, this is the dominant configuration. However, it is of course possible to select kinematic configurations in

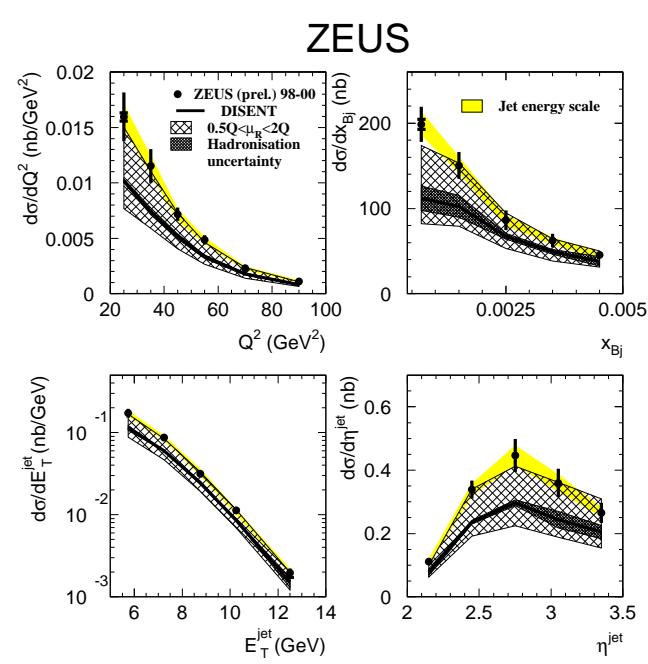

Figure 17. Forward jet cross sections at HERA.

which a large evolution in $x$ (or equivalently in rapidity) is required, but where this evolution takes place at a $\mathrm{Q}^{2}$ which is both in the perturbative regime and approximately constant. New measurements have been made in forward jet production (Fig.17) in DIS and other related processes at HERA ${ }^{38}$.

In such a region the DGLAP evolution is not applicable. Thus if NLO fixed order QCD with DGLAP parton densities is used to try and predict such cross sections, the predictions have large uncertainties. It is also seen that they usually lie below the data. Leadinglogarithmic Monte Carlos can do better than this, and in particular, the CCFM-based MC CASCADE $^{37}$ probably has the ability to describe such cross sections. However, it has a strong dependence on the unintegrated gluon density, which is extracted from fits to data. The new data should be used to constrain this further.

Such effects may also be studied in vector meson and photon production. The vector mesons I leave to the next speaker ${ }^{2}$, but will mention here the new data from DELPHI $\gamma^{*} \gamma^{*}$ collisions, where a signifcant $x$ evolution can occur along the exchanged quark line. 
Again, calculations (BFKL-based) which resum $\log (x)$ terms seem to have the best chance of describing the data.

A consistent, and reasonably precise, description of high rapidity/low $x$ data seems to be within reach. This would give a real boost to the credibility of this approach, and would be a great help for predicting forward jet rates at LHC.

\section{Conclusion}

In an increasing number of important processes at high energy colliders, perturbative QCD calculations, and the data, are rather precise, and in rather good agreement with each other. New data from Tevatron and HERA, and (re)analysis of old data from PETRA and LEP, continue to improve the situation, as do theoretical advances. There is still room for improvement of course, but for some important processes QCD is now very precisely understood, and there have been recent significant advances in measurement and theory. As an aside, the point is now being reached where for some observables, electroweak effects are comparable to QCD uncertainties $^{39}$. For other processes, while QCD is becoming better understood, there is still experimental and theoretical work to do. A list of such processes, in approximate decreasing order of how well they are understood, could be:

- Parton density functions at high $\mathrm{Q}^{2}$ and intermediate $x$, ideal jet fragmentation.

- Multijet processes, Boson+jets; Heavy flavour production.

- Parton density functions at low and high $x$.

- High rapidities and rapidity gaps.

- Diffraction, absorption and total cross sections.
- Off-diagonal and unintegrated parton density functions.

- Underlying events (a topic hardly touched on here, but where there is lots of work on tuning to Tevatron, HERA, SPS and other data $\left.{ }^{40,41}\right)$.

In all these areas existing data, as well as data still to come from Tevatron run II, HERA II and RHIC, provide a challenge. Data from LHC will make great use of such developments, and will also challenge the theory further.

\section{Acknowledgments}

My thanks to D. Alton, R. Field, G. Ingelman, E. Perez, G. Salam, J. Schiek, P. Wells and M. Wing for discussions and material, as well as to those others whose work I have presented here. I also thank the organisers for a conference which was stimulating, well run and fun.

\section{References}

1. L. Rolandi, F. Gianotti, these proceedings.

2. G. Ingelman, these proceedings.

3. ZEUS Coll., ZEUS-prel-05-016; LP2005 paper 283.

4. OPAL Coll., G. Abbiendi et al., Phys. Rev. D 69032002 (2004). [arXiv:hepex/0310048].

5. DELPHI Coll., M. Seibert, K. Hamacher and J. Drees DELPHI-2005-12 CONF 732; LP2005 paper 135.

6. H1 Coll., A. Aktas et al. Eur. Phys. J. C 38447 (2005) [arXiv:hep-ex/0408149]; H1prelim-05-074, LP2005 paper 407.

ZEUS Coll., S. Chekanov et al. [arXiv:hepex/0508019]; ZEUS-prel-05-007, LP2005 paper 266.

7. L3 Coll., P. Achard et al. Phys. Lett. $B 554105$ (2003) [arXiv:hep-ex/0301025]. Phys. Lett. B 602157 (2004) [arXiv:hepex/0410012].

8. DELPHI Coll., M. Chapkin et al., DELPHI2005-018 CONF 738. LP2005 paper 90. 
9. H1 Coll., H1prelim-05-074, H1prelim-05077; LP2005 papers 405, 409.

10. ZEUS Coll., S. Chekanov et al., [arXiv:hepex/0505008];

[arXiv:hep-ex/0507089]; ZEUS-prel-04-024, LP2005 paper 265.

11. For a recent review, see J. M. Butterworth and M. Wing, "High energy photoproduction", Rep. Prog. Phys. 68 2773-2828 (2005) [arXiv:hep-ex/0509018].

12. H1 Coll., A. Aktas et al., [arXiv:hepex/0507081]; Eur. Phys. J. C 40349 (2005) [arXiv:hep-ex/0411046].

13. T. Sjöstrand et al. Comput. Phys. Commun. 135238 (2001) [arXiv:hep$\mathrm{ph} / 0010017]$.

14. G. Corcella et al., JHEP 0101010 (2001) [arXiv:hep-ph/0011363].

15. S. Frixione and B. R. Webber, JHEP $0206 \quad$ (2002) [arXiv:hep-ph/0204244];

S. Frixione, P. Nason and B. R. Webber, JHEP 0308007 (2003) [arXiv:hep$\mathrm{ph} / 0305252]$.

16. J. M. Butterworth, J. R. Forshaw and M. H. Seymour, Z. Phys. C 72637 (1996) [arXiv:hep-ph/9601371].

17. CDF Collaboration Note 6985, available from http://www-cdf.fnal.gov

18. D0 Collaboration D0 note 4754-CONF.

19. DELPHI Coll., P. Bambade et al, LP2005 paper 57.

20. JADE Coll., C. Pahl, et al [arXiv:hepex/0408123]; J. Schieck, et al [arXiv:hepex/0408122]. H1 Coll., H1prelim-05-033, LP2005 paper 390.

ZEUS Coll., S. Chekanov et al. arXiv:hepex/0502007; LP2005 paper 281, ZEUS-prel05-024

OPAL Coll, G. Abbiendi et al., Eur. Phys. J. C 40287 (2005) [arXiv:hep-ex/0503051]; [arXiv:hep-ex/0507047].

21. ALEPH Coll., S. Schael et al., [arXiv:hepex/0506072];

M. Davier, A. Höcker and Z. Zhang, [arXiv:hep-ph/0507078].

22. H1 Coll., A. Aktas et al., Eur. Phys. J. C 38437 (2005) [arXiv:hep-ex/0407018]. D0 Coll., D0-4859-CONF.

23. CDF Collaboration, D. Acosta et al., Phys. Rev. Lett. 95022003 (2005) [arXiv:hepex/0412050].

24. D0 Coll., V. M. Abazov et al. Phys. Rev. Lett. 94221801 (2005) [arXiv:hep- ex/0409040].

25. S. Schumann, LP2005 poster 50.

T. Gleisberg et al, JHEP 0402056 (2004), [arXiv:hep-ph/0311263].

26. T. Binoth et al., Eur. Phys. J. C 16311 (2000) [arXiv:hep-ph/9911340].

27. C. Balazs, E. L. Berger, S. Mrenna and C. P. Yuan, Phys. Rev. D 576934 (1998) [arXiv:hep-ph/9712471].

28. D0 Coll., "Measurement of $Z \rightarrow e^{+} e^{-}$rapidity distribution", http://wwwd0.fnal.gov/Run2Physics/WWW/results/ew.htm

29. D0 Coll., D0-4794-CONF.

30. G. Salam, these proceedings.

31. C. Diaconu, these proceedings.

32. ZEUS Coll., ZEUS-prel-05-005, LP2005 paper 261.

33. CDF Coll., D. Acosta et al. Phys. Rev. D 71051104 (2005) [arXiv:hep-ex/0501023].

34. H1 Coll., A. Aktas et al., Phys. Lett. B 598 159 (2004) [arXiv:hep-ex/0406029];

H1prelim-04-042, LP2005 paper 389.

35. ZEUS Coll., S. Chekanov et al., Eur. Phys. J. C42, 1 (2005), [arXiv:hep-ph/0503274].

36. C. Gwenlan, A. Cooper-Sarkar and C. Targett-Adams, [arXiv:hep-ph/0509220], prepared for the HERA-LHC workshop.

37. H. Jung and G. P. Salam, Eur. Phys. J. C 19351 (2001) [arXiv:hep-ph/0012143].

38. ZEUS Coll., LP2005 paper 25, 278. H1 Coll., LP2005 paper 378, 379, 381, 384.

39. E. Maina et al., LP2005 paper 359; A. D. Martin, R. G. Roberts, W. J. Stirling and R. S. Thorne, Eur. Phys. J. C 39155 (2005) [arXiv:hep-ph/0411040].

40. HERA-LHC workshop, http://www.desy.de/ heralhc/

41. TEV4LHC workshop, http://conferences.fnal.gov/tev4lhc/ 